\title{
Improving Multipath Data Delivery and Energy Efficiency in Few Disjoint Paths Scenario
}

\author{
Rehan Qayyum*, Junaid Ahmed Khan ${ }^{\dagger}$ and Adnan Iqbal ${ }^{\ddagger}$ \\ ${ }^{* \dagger}$ School of Electrical Engineering and Computer Science, National University of Sciences and Technology, H-12, Islamabad. \\ ${ }_{\ddagger}^{\ddagger}$ Iqra University, Islamabad Campus, 5, Khayaban-e-Johar, H-9, Islamabad. \\ $\left\{{ }^{*} 10 \mathrm{mseerehan},{ }^{\dagger} 10 \mathrm{mse}\right.$ junaidk $\} @$ seecs.edu.pk, ${ }^{\ddagger}$ adnan.iqbal@iqraisb.edu.pk
}

\begin{abstract}
Multipath data transmission is an established mechanism to ensure reliable data delivery in mission critical sensor networks. Advantages of a multipath data delivery protocol can be realized if there exist large number of paths in the network. However, this is generally not the case as finding alternative paths is an expensive process. Therefore, we propose minor modifications to a basic multipath approach so that it does not require large number of multipaths. We augment multipath approach with only one per hop retransmission. Through analytical models and trace driven simulations, we show that the data delivery probability of the proposed approach is generally higher than the multipath approach. We also show that the proposed approach consumes upto $50 \%$ less energy than the basic multipath approach.
\end{abstract}

\section{INTRODUCTION}

Reliable delivery of data is a fundamental requirement in Wireless Sensor Networks (WSN). Various approaches have been proposed in the past for ensuring reliability including link layer retransmissions and forward error correction (FEC) . Multipath transmission is also considered a suitable alternative for retransmissions and FEC. The basic idea in each approach is to achieve reliability through redundancy. The redundancy to achieve reliable data delivery improves packet delivery but also creates significant overhead. Wireless sensor networks have very limited resources and energy efficiency is a fundamental constraint in protocol design. Therefore, a reliable data delivery protocol for wireless sensor network aims to achieve two conflicting objectives of high data delivery and high energy efficiency.

On the other hand, WSN devices are prone to failure, resulting into failure of an end-to-end path considered by data source as available. Multipath approach is the only viable solution in such scenarios and is therefore in practice to achieve this objective [1] [2] [3]. In multipath transmission replicas of the data are transmitted on a number of available multipaths. This approach serves good, however it suffers from several problems in its basic form. In an error-prone network, several multipaths are required to achieve acceptable data delivery rates. However, unavailability of large number of multiple disjoint path in a network [4] limits number of effective multipaths to be used. This observation necessitates the requirement of modifying multipath transmission such that it does not require to have non-practical large number of disjoint paths and still caters for the node failure scenario.
This paper presents one such approach, in which we use only two multipaths and augment this approach with only one link layer retransmission.

In this paper we first present general analytical models for retransmissions over a single path and transmissions on multiple paths. We then present results of this modeling and identify why it is a plausible idea to combine both the approaches. We then describe our proposed scheme, in which we use a multipath approach restricted to very few disjoint path and makes use of limited number of link layer transmissions. We evaluate all three schemes using bit-error traces collected over working wireless sensor network. Results of this analysis reveals that proposed scheme provide upto $100 \%$ improvement in end-to-end packet delivery probability and $50 \%$ decrease in energy consumption for different size path lengths.

Rest of the paper is organized as follow: In Section II, we describe the basic modeling of retransmissions and multipath approaches followed by Section III, which covers proposed scheme. Section IV describes empirical end-to-end packet delivery evaluation results in detail. Section V covers energyefficiency analysis and Section VI discusses some relevant work. Finally we conclude our major findings in Section VII

\section{Problem with Multipath and Possible IMPROVEMENT}

In Ad hoc networks, multipath data delivery approach is being used frequently and several routing protocols such as AOMDV [1], MP-DSR [2], MSR [3] have been proposed to support multipath approach.

In a nutshell, multipath routing protocols find more than one disjoint paths from source to destination and transmit the same data frame to the same destination through these different paths. Negative and positive acknowledgments of individual frames are not allowed at link layer for the sake of simplicity.

The biggest advantage of multipath approach is to increase overall reliability of data delivery even though some nodes fail during data delivery operation, since alternate paths exist and can be used for data delivery. Such a facility is missing in other approaches - such as retransmissions on a single path. However, we argue that multipath approach when compared to unicast approach with retransmissions, does not provide 
similar data delivery probability as that of unicast with retransmission.

If we consider that source $(s)$ to destination $(d)$ pair in a network has $N$ disjoint paths and frame error rates on each hop in a network are independent, then the end-to-end packet delivery probability from $s$ to $d$ for an abstract multipath data delivery protocol can be defined as

$$
P_{m, N}=1-\prod_{j=1}^{N}\left\{1-\prod_{i=1}^{m} p_{j, i}\right\}
$$

where $p_{j, i}$ means packet delivery probability on $i-t h$ hop in $j-t h$ path. Number of hops on each path are considered same and equal to $m$. This is a relaxing assumption, since in real networks higher order multipaths gradually become longer and more error prone - than initial shortest paths.

Just like any other reliable data delivery protocol, above equation emphasizes the fact that increasing redundancy number of redundant paths - will increase data delivery probability. However, this equation becomes more meaningful when we compare it with unicast retransmission based approach.

If $R$ retransmissions are allowed per hop, then the end-toend packet delivery probability from $s$ to $d$ on a unicast path can be defined as

$$
P_{m, R}=\prod_{i=1}^{m}\left\{\sum_{j=1}^{R}\left(1-p_{i}\right)^{j-1}\left(p_{i}\right)\right\},
$$

where $p_{i}$ means packet delivery probability on $i-t h$ hop. Total number of hops is $m$ and allowed transmissions are denoted by $R$.

Figure 1 shows a comparative result of equations 1 and 2, where we have equated number of redundant paths $N$ with number of transmissions $R$ on a unicast path. For the ease of understanding, we have considered a fixed frame error rate on each hop. This Figure gives us some insight and clarifies that retransmissions on a unipath are more reliable than using a similar number of multipaths. However, further understanding of this phenomenon can be achieved by examining Figure 2 .

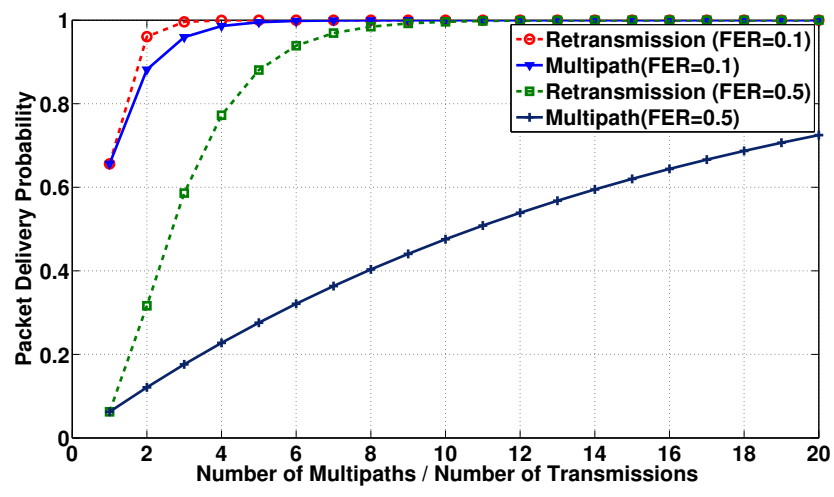

Figure 1. Analytical End-to-end Packet Delivery Probability Comparison of Multipath and Retransmissions

Figure 2 plots the difference between equations 1 and 2 for same parameters as considered in Figure 1. We can see from
Figure 2 that the difference between unicast and multipath transmission is maximum in initial few transmissions/paths. For instance, in FER $=0.3$, difference increase until transmission/multipath $=4$ and after that it starts decreasing.

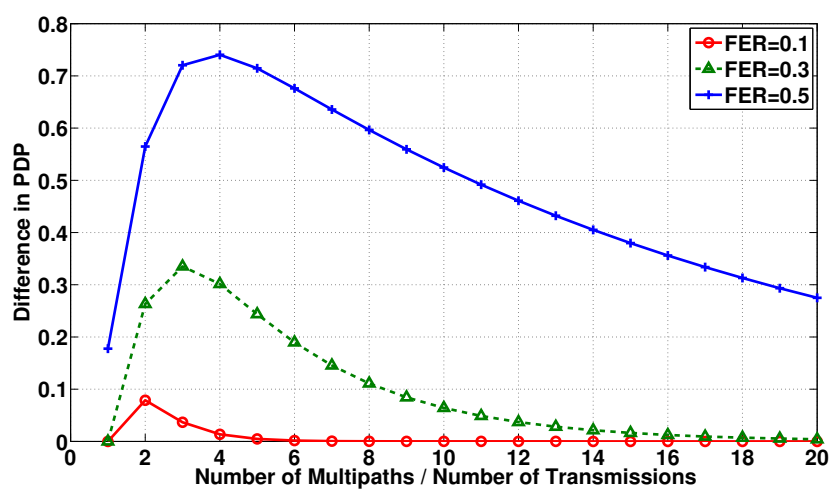

Figure 2. Analytical End-to-end Packet Delivery Probability Difference Plot

This leads us to hypothesis that if we develop a hybrid data delivery approach, which is a combination of both multipath and retransmissions, may provide better performance. In next section, we describe one such possible scheme.

\section{PRoposed SCHEME}

Let us consider a source node $s$ and a destination node $d$. We define a hybrid multipath data delivery protocol which allows to make use of a maximum of $N$ disjoint paths and also allows a maximum of $R$ transmissions on each hop. Then end-to-end packet delivery probability can be defined as:

$$
P_{m, N, R}=1-\prod_{j=1}^{N}\left\{1-\prod_{i=1}^{m}\left\{\sum_{k=1}^{R}\left(1-p_{j, i}\right)^{k-1}\left(p_{j, i}\right)\right\}\right\}
$$

where $p_{j, i}$ means packet delivery probability on $i-t h$ hop in $j-t h$ path. As in previous section, total number of hops per path are considered same and equal to $m$. Number of allowed transmissions are denoted by $R$.

This scheme is perfectly plausible, since there exists several algorithms to compute multiple paths between a source and a destination [5] [2] and retransmissions are hop-by-hop only. However, it may not be practical to introduce large number of per-hop transmissions since it may ensue greater complexity. So the idea is to restrict number of retransmissions to a minimum. Through the analysis of equations 1 and 3, we found that adding only one retransmission while having only two paths results into better performance than a simple multipath approach containing 5 paths.

We compared Equation 3 using one retransmission and two multipaths with Equation 1 using 5 multipaths and 10 multipaths. This comparison is shown in Figure 3. We have considered the same frame error rate on each hop and also considered the same number of hops on each of the disjoint paths. This Figure clearly shows that our proposed scheme results in a better packet delivery probability than the multipath 


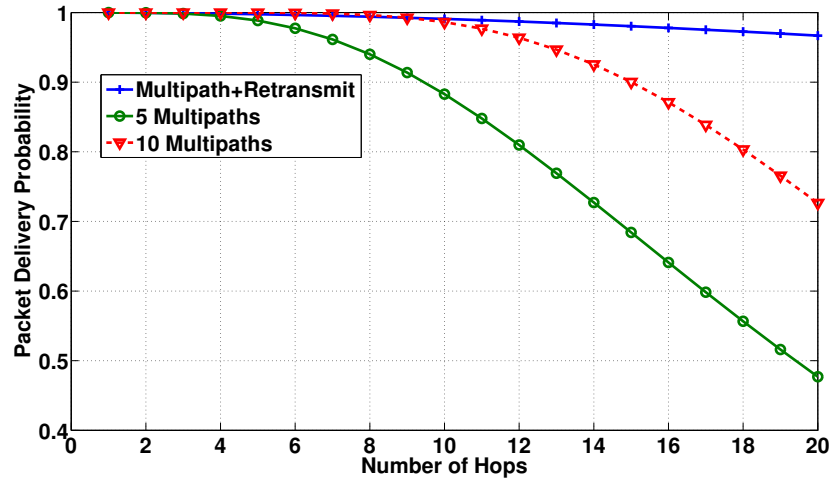

Figure 3. Theoretical Comparison of End-to-End Packet Delivery Probability of Multipath and Proposed Scheme

scheme even with as large as 5 multipaths. For other series in the plot - 10 multipaths - simple multipath approach is slightly better when path length is small. However, as the path length increases, packet delivery probability of proposed scheme appears better than simple multipath approach.

We have already shown in Figure 2 that we need to design a scheme which covers the performance difference of pure retransmission based approach pure multipath based approach for initial few transmissions and multipaths. Considering this observation along with discussion in this section, it is evident that only few multiple paths are needed. In fact, in next section, we show through trace driven simulation that allowing only one retransmission and only one redundant path has consistently better performance than that of using 5 multipaths with no retransmission.

\section{End-TO-End PACKET DELIVERY ANALYSis}

To validate findings of previous sections, we have collected large number of traces from working sensor network testbed. This section outlines collection of traces, methodology for analytical and empirical analysis and finally discusses evaluation results. First, we briefly discuss collection of traces.

\section{A. Data Collection}

We used Crossbow's Micaz motes (now available from MEMSIC)[6] to collect residual bit-error traces. Traces were collected using four different network setups with a fixed basestation receiver and four different transmitters located at distances of $5-12$ meters from the transmitter. The senders transmitted fixed-sized 20-byte frames at a rate of 10 frames per second. For each experiment , 6-7 traces were collected. We have used 20 traces from these collected traces to mimic a path length of 20 -hops. These 20 traces are picked randomly. The bit-error rate and frame-error rate for each trace are given in Table I. Further detail of these traces is available in [7].

Since our objective is to estimate the performance of our proposed scheme and simple multipath scheme, we need large number of disjoint paths with similar path characteristics, leading us to have more traces than actually collected. Therefore, we generated number of synthetic traces using 2-Tier Markov
Table I

TraCE SEQuence For Simulation

\begin{tabular}{|c|c|c|c|}
\hline Hop No. & Trace & Bit Error Rate & Frame Error Rate \\
\hline 1 & PhdLab_3_8 & 0.000962 & 0.02813 \\
\hline 2 & Up_Floor_2_12 & 0.000314 & 0.01187 \\
\hline 3 & OutRoom_2_5 & 0.001007 & 0.05351 \\
\hline 4 & Stair_7_4 & 0.000533 & 0.02473 \\
\hline 5 & OutRoom_3_8 & 0.001046 & 0.03534 \\
\hline 6 & PhdLab_2_7 & 0.004332 & 0.14130 \\
\hline 7 & Stair_6_5 & 0.001258 & 0.04808 \\
\hline 8 & OutRoom_4_7 & 0.001158 & 0.04726 \\
\hline 9 & PhdLab_4_7 & 0.015772 & 0.51708 \\
\hline 10 & Stair_4_5 & 0.002408 & 0.06695 \\
\hline 11 & Up_Floor_3_12 & 0.000253 & 0.01044 \\
\hline 12 & OutRoom_1_5 & 0.000015 & 0.00049 \\
\hline 13 & Up_Floor_4_12 & 0.000102 & 0.00368 \\
\hline 14 & OutRoom_5_8 & 0.000216 & 0.00839 \\
\hline 15 & Up_Floor_5_12 & 0.000121 & 0.00474 \\
\hline 16 & OutRoom_7_5 & 0.000014 & 0.00061 \\
\hline 17 & Up_Floor_6_12 & 0.000092 & 0.00340 \\
\hline 18 & Stair_3_5 & 0.000437 & 0.01703 \\
\hline 19 & Up_Floor_7_12 & 0.000437 & 0.02416 \\
\hline 20 & PhdLab_1_8 & 0.011436 & 0.35520 \\
\hline & & & \\
\hline
\end{tabular}

synthetic trace generator. We used Gilbert channel at frame level and a $3-r d$ order Markov chain at bit level. This method is shown to be statistically accurate as compared to other approaches in [7]. For each trace collected from operational network, 10 synthetic traces were generated. Each synthetic trace is of size 10000 frames. We performed evaluation for different network topologies - varying number of nodes in a unique path and varying number of multiple disjoint paths. Now we discuss evaluation methodology and results.

\section{B. Analytical Evaluation}

For analytical evaluation, we derived FER from traces and used equations 1,2 and 3 to compute the values of end-to-end packet delivery probability. For simple multipath approach, we calculated end-to-end packet delivery probability for a minimum of 2 multipaths to a maximum of 20 multipaths. Similarly, we varied number of hops in a unique path from 2 to 20 . Figure 4 describes a subset of these results. Other results depict similar trends and are skipped for brevity sake.

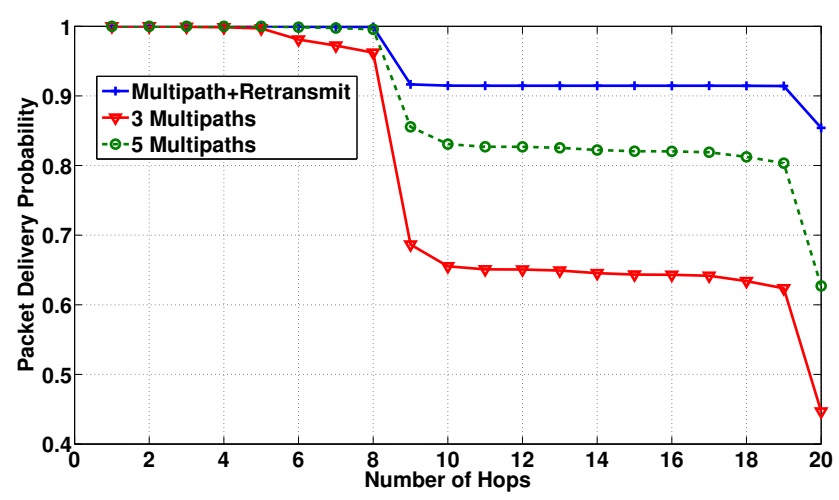

Figure 4. Analytical Comparison of Packet Delivery Probability of Multipath and Proposed Scheme 
Figure 4 plots analytical packet delivery probability using 3 multipaths, 5 multipaths and our proposed scheme with 2 paths and 1 per hop retransmission. Every point in the plot is obtained for a different path length mentioned on x-axis.

Figure 4 identifies number of trends. First, it shows that for small number of hops, there is not much difference between simple multipath and the proposed scheme. Second, it also shows that at some hops there is notable decrease in the endto-end probability - such as at hop 5 and 9 . This is because at these hops, FER is fairly higher than average (at hop 9 FER is 0.35). Third, we consistently find that using proposed approach is better than using simple multipath for large path lengths. For instance, for path length 20 hops, using 5 different multipaths results into a packet delivery probability of 0.62 , where as using 1 retransmission and only 1 redundant path provides packet delivery probability of 0.85 .

This theoretical analysis suggests that using a combination of 1 retransmission and 1 extra path is better than simple multipath approach. We further investigate this claim in next sub-section using trace driven simulations.

\section{Empirical Evaluation}

Using collected and generated traces, we have simulated simple multipath scheme for different number of multipaths and a hybrid of both with varying number of paths and transmissions. Just like analysis in the previous sub-section, we have calculated end-to-end packet delivery probability for a minimum of 2 multipaths to a maximum of 20 multipaths. Similarly, we varied number of hops in a unique path from 2 to 20 . Each hop was represented by a unique trace file. In every simulation, we transmitted at least 77,000 unique packets from source to destination. Packet delivery probability is calculated as the ratio of unique packets received at destination to the actual uniques packets transmitted by the source. In case of multiple deliveries at the same packet at destination, duplicates are discarded. Figure 5 describes a subset of these results. Other results depict similar trends and are skipped for brevity sake.

Figure 5 covers three scenarios, multipath with 3 and 5 alternate paths, and proposed scheme with 1 retransmission and only 2 alternate paths. Figure 5 depicts similar trends like analytical evaluation in previous sub-section. We find that a combination of 1 retransmission and 2 multipaths is consistently better than 5 multipaths for all topologies considered in this analysis. Smaller path lengths have very little difference in packet delivery probability whereas in larger path lengths the difference between multipath and the proposed approach is become larger. For instance, for hop length 20 the difference is greater than $11 \%$.

These empirical results conform to the theoretical results of previous section except for the fact that difference between two approaches is smaller in empirical evaluation. It is obvious from this analysis that the proposed scheme is a viable approach. We further strengthen this claim by exploring energy efficiency performance of both the approaches in next section.

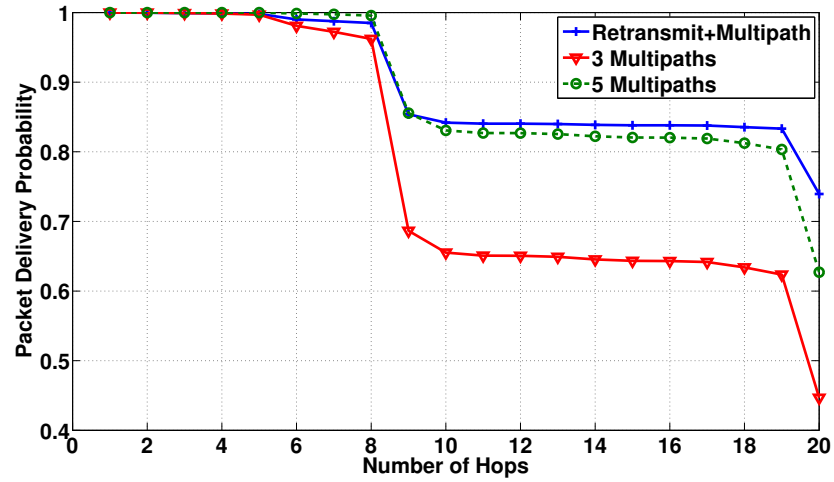

Figure 5. Empirical Evaluation of End-to-end Packet Delivery Probability

\section{ENERGY-EFFICIENCY ANALYSIS}

Energy efficiency is a key design issue in energy-constrained wireless sensor networks that needs to be addressed in order to increase the network lifetime. We have performed empirical energy efficiency analysis of our proposed scheme and simple multipath scheme using a measure of number of per hop transmissions for a unique packet delivery from source to end node. All simulations are carried out using same topologies as in previous section. In each simulation, we transmit a minimum of 77,000 unique frames and record number of transmissions of the same packet on each hop in every path. At the end of simulation, we calculate average number of per hop transmissions for a unique packet delivered to the destination by dividing total number of per hop transmissions with total number of unique packets delivered to the destination. Duplicate packets received at the destination are counted as only one successful delivery.

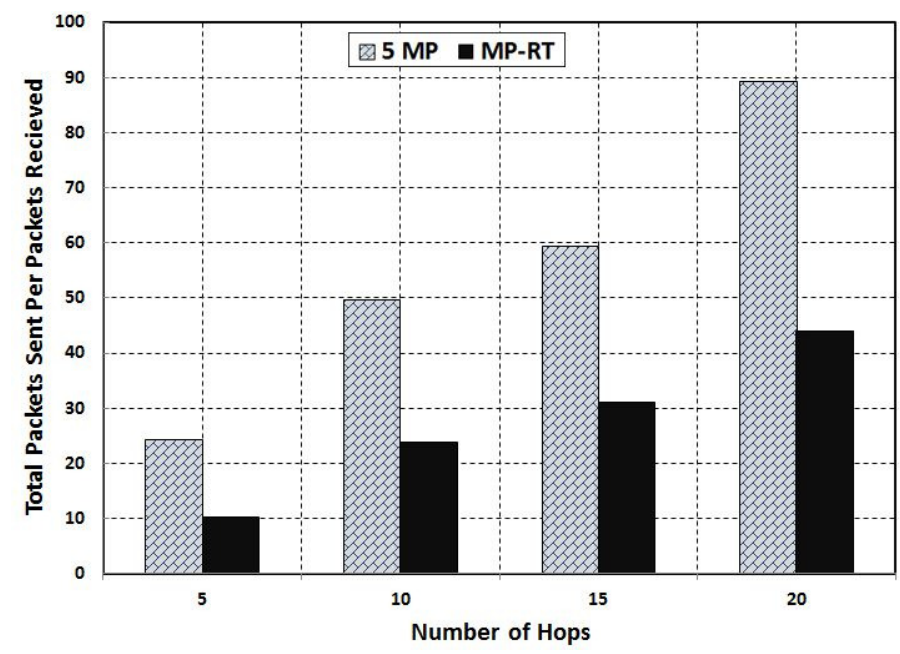

Figure 6. End-to-end Energy-efficiency Analysis

Figure 6 shows a comparison between proposed scheme with 5 multipath scheme for energy efficiency. A number of trends are identified in Figure 6. First, it shows that with an increase in number of hops the total packets sent per 
each unique delivered packet increases for both schemes. Second, it shows that our proposed scheme is consistently better. For instance, proposed scheme requires $6,16,31$ and 44 per hop transmissions for a single packet delivery on a network of $5,10,15$ and 20 hops respectively. For a similar network, multipath with 5 paths require 24, 49, 59 and 89 per hop transmissions. These results suggest that the proposed approach has not only higher packet delivery probability but also has better energy utilization.

Since multipath is in use for quite sometime now. It is appropriate to describe some related effort. In next section, we outline some important studies in this domain.

\section{RELATED WORK}

Several models have been developed to address the issue of reliability in sensor networks. Most of the recent efforts to our knowledge are mainly focused on computing multiple paths for transmission out of which the most common are MP-DSR [2] and AOMDV[1]. In MP-DSR, the existing routing protocol DSR [8] is modified to discover multiple disjoint paths and transmit data on these disjoint paths. The application sending a route request sets an end-to-end reliability requirement in its route discovery message. MP-DSR assures that each disjoint path discovered must meet the desired end-to-end reliability requirement. AOMDV is proposed by incorporating multipath scenario in the existing AODV[9] protocol. In AOMDV, a source node computes multiple disjoint paths in a single route discovery message to its desired destination. The aim is to assure multiple loops-free and link-disjoint paths are computed. For this purpose an additional attribute - advertised hop count - is introduced to the existing AODV protocol. It is then recommended to use link-disjoint paths instead of nodedisjoint paths for route discovery messages.

Both MP-DSR and AOMDV protocols are designed for Wireless Ad-hoc networks and are not feasible to implement in sensor network due to the power constraints associated with sensor networks.Efforts specific to sensors networks have been developed recently, one such approach is proposed by Gou and Yoo in [10]. This scheme focuses on finding multipaths in a sensor network using a hop cost based optimization model.

Saleem et al. [11] has proposed a modification to existing On-demand flooding based routing protocols by considering the use of multiple disjoint paths to ensure packet delivery reliability. They have recommended to use a small set of node disjoint paths instead of computing total number of available disjoint paths. They have shown that increasing the number of paths beyond a certain level yields minimal improvements in packet delivery reliability.

Most of the above mentioned protocols focus on computing multipaths using different methods. However, there is very little effort towards the improvement of basic multipath approach. Therefore, in this paper, we have proposed an approach which targets basic multipath approach and suggests minor changes which result into end-to-end packet delivery probability and energy efficiency.

\section{CONCLUSION AND FUTURE WORK}

In this paper we first established that multipath data delivery can be improved. Then we presented a hybrid approach that does not require large number of multiple disjoint paths. Theoretical and empirical analysis of the proposed approach provided better end-to-end packet delivery probability as compared to simple multipath approach. In future, we plan to develop detailed stochastic models for the proposed approach that also involve reliability and energy efficiency. We further plan to test this protocol through a real testbed implementation.

\section{REFERENCES}

[1] M.K. Marina and S.R. Das. Ad hoc on-demand multipath distance vector routing. Wireless Communications and Mobile Computing, 6(7):969-988, 2006.

[2] R. Leung, J. Liu, E. Poon, A.L.C. Chan, and B. Li. MP-DSR: a QoS-aware multi-path dynamic source routing protocol for wireless ad-hoc networks. In Local Computer Networks, 2001. Proceedings. LCN 2001. 26th Annual IEEE Conference on, pages 132-141. IEEE, 2001.

[3] L. Wang, L. Zhang, Y. Shu, and M. Dong. Multipath source routing in wireless ad hoc networks. In Electrical and Computer Engineering, 2000 Canadian Conference on, volume 1, pages 479-483. Ieee, 2000.

[4] Z. Ye, S.V. Krishnamurthy, and S.K. Tripathi. A framework for reliable routing in mobile ad hoc networks. In INFOCOM 2003. Twenty-Second Annual Joint Conference of the IEEE Computer and Communications. IEEE Societies, volume 1, pages 270-280. Ieee, 2003.

[5] S.J. Lee and M. Gerla. Split multipath routing with maximally disjoint paths in ad hoc networks. In Communications, 2001. ICC 2001. IEEE International Conference on, volume 10, pages 3201-3205. IEEE, 2001.

[6] MEMSIC: Wireless Modules, IRIS, MicaZ, TelosB, Imote2, Cricket. http://http://www.memsic.com/products/ wireless-sensor-networks/wireless-modules.html.

[7] A. Iqbal and S.A. Khayam. Accurate residual wireless channel simulation using a two-tier Markov model. SIMULATION, 2012.

[8] D.B. Johnson, D.A. Maltz, J. Broch, et al. DSR: The dynamic source routing protocol for multi-hop wireless ad hoc networks. Ad hoc networking, 5:139-172, 2001.

[9] C.E. Perkins and E.M. Royer. Ad-hoc on-demand distance vector routing. In Mobile Computing Systems and Applications, 1999. Proceedings. WMCSA'99. Second IEEE Workshop on, pages 90-100. IEEE, 1999.

[10] H. Gou and Y. Yoo. A Low Cost Reliable Data Transmission Protocol over Multipath in Wireless Sensor Networks. In 2012 26th IEEE International Conference on Advanced Information Networking and Applications, pages 810-816. IEEE, 2012.

[11] M. Saleem, I. Ullah, S.A. Khayam, and M. Farooq. On the reliability of ad hoc routing protocols for loss-anddelay sensitive applications. Ad Hoc Networks, 9(3):285299, 2011. 